RESEARCH REPORT

\title{
The demographic and social class basis of inequality in self reported morbidity: an exploration using the Health Survey for England
}

\author{
S Asthana, A Gibson, G Moon, P Brigham, J Dicker
}

J Epidemiol Community Health 2004;58:303-307. doi: 10.1136/jech.2002.003475

See end of article for authors' affiliations

.....................

Correspondence to: Professor G Moon, School of Social and Historical Studies, University of Portsmouth, Milldam, Burnaby Road, Portsmouth POI 3AS, UK; graham. moon@port.ac.uk

Accepted for publication 29 May 2003
Study objectives: To assess the relative contribution of age and social class to variations in the prevalence of a selection of self reported health problems. To examine the implications of observed variations for research on health inequalities.

Design: Secondary analysis of the Health Survey for England (1991-1997) using morbidities that are particularly prone to class effects. A statistical measure of the "relative class effect" is introduced to compare the effects of adjusting for social class and age.

Main results: There is substantial variation in the relative importance of the age and class distributions of different diseases. Age effects often overshadow those of class even for conditions where an apparently strong social gradient exists. Only for self reported mental health among women does the social gradient exceed the age gradient. Within the context of a dominating age gradient, social gradients are relatively high for mental health and general health for both sexes. Variation in the relative strengths of the social gradients between the sexes are observed for angina symptoms.

Conclusions: Given variations in the "relative class effect", analysis recognising the distinct contributions of age, sex, and social class to specific morbidities is advocated as a transparent and robust approach to the assessment of morbidity based inequality.
T here has been a longstanding recognition of the persistent relation between low socioeconomic status and higher mortality rates in England. ${ }^{1}$ Less consideration has been given to morbidity based socioeconomic inequalities but the evidence remains equally compelling. ${ }^{2-4}$ Overall, it is impossible to deny that there are consistent socioeconomic gradients to both mortality and morbidity. Studies of these gradients usually conceptualise mortality or morbidity as age sex standardised rates or ratios and lower socioeconomic groups generally do less well.

This paper examines gradients in self reported morbidity in England. It aims to assess the relative roles of age, sex, and social class as factors underpinning these gradients. The hypothesis underpinning our aim is methodological in origin. We contend that the emergence of a large body of literature on social differentials in morbidity has shifted attention away from the influence of demography (age and sex) on the prevalence of specific diseases. In part this emphasis on social differentials may stem from a tendency to "control out" age and sex through standardisation processes before examining relations with socioeconomic status. Our initial hypothesis is thus that socioeconomic gradients in morbidity are less than those associated with age.

The evidence for social class gradients in self reported morbidity is substantial. To ensure direct relevance to the concerns of this paper we limit our study to studies based in England and, where possible, to research that has used the UK registrar general's social class classification and focused on self reported morbidity. Studies were identified using a Medline search for the years 1984-2002.

The evidence for strong associations between high morbidity and low social class is replicated in individual analyses of large routine national sources, ${ }^{5-7}$ research using local surveys, ${ }^{3}$ and aggregate area based studies. ${ }^{4}$ It is also replicated for a wide range of conditions. Conditions where gradients are common to both sexes include psychiatric morbidity, $^{79}$ heart disease, ${ }^{34610-13}$ cerebrovascular disease, ${ }^{346}$ self reported asthma, ${ }^{34-16}$ and diabetes. ${ }^{17} 18$ Diseases where a sex consistent inverse association seems to exist between prevalence and low social class include complaints of the bowel/colon ${ }^{19}{ }^{20}$ and hay fever. ${ }^{21}$

There are important caveats to this picture of consistent gradients. Firstly, there are pronounced variations by gender with gender based inequality remaining after controlling for social status ${ }^{22}$ and social gradients being flatter for women. ${ }^{23}$ It is clear that inequalities need to be assessed separately for men and women. ${ }^{24}$ Secondly, gradients vary by age. Elderly women assess their health less positively than men and class based inequality is important in old age. ${ }^{25}$ Conversely, social inequalities in health may be marked in infant populations but among young people there is often little class difference. ${ }^{26}$ Class re-emerges as a significant factor in populations aged over 20, is clearly present by the age of 35, and generally increases with age. 232728 Thirdly, the detailed pattern of relations between class and health show considerable diversity. ${ }^{29}$ Thus, for some conditions, gradients may be consistent with age. For others, gradients steepen or flatten with age, or relate to sex but not age.

The balance of evidence in the existing literature thus points to an expectation that the association of social class and health will vary with sex and vary significantly with age but not in a uniform fashion. The limited evidence available to date on the contribution of class to inequalities in health after controlling for non-class attributes, makes the case for the importance of gender. ${ }^{30}$

\section{METHODS}

To explore the strength of social gradients relative to demographic variations in morbidity, data were abstracted from the 1991-1997 runs of the Health Survey for England. This annual official survey has reported on a wide range of "self reported longstanding illnesses" and has had an annual 
sample size that rose from about 7000 adults in its first run to about17 000 by 1997. Its adult response base represents roughly $75 \%$ of potential respondents and is representative of England in terms of age, sex, socioeconomic status, and region. ${ }^{6}$ In addition to information on self reported longstanding illnesses, the survey includes a variety of individual and contextual variables. For the purposes of this study we have focused on age (16+), sex and social class defined, as in the 1991 UK census, by the occupation of the head of the household. Though other measures and definitions of social class exist and are often methodologically preferable, this is the standard definition historically used in UK studies of class based health inequalities. ${ }^{1}$ We limit our analysis to self reported morbidities that demonstrate separate significant $(\mathrm{p}<0.01)$ associations with poor social class for both men and women respondents to the 1991-1997 runs of the Health Survey for England.

For each condition, prevalence tables were produced for men and women comprising seven age categories (16-24; 10 year bandings to 74 , and $75+$ ) and the standard six social class categories (ranging from $\mathrm{I}=$ highest to $\mathrm{V}=$ lowest with III subdivided into manual and non-manual). The numerator in each cell is the number of respondents in each category who are recorded as suffering from a particular condition. The denominator is the total number of respondents in each category who provided a valid response to the relevant question. The prevalence for each condition is then expressed as a rate per 10000 of the denominator. To ensure a sufficiently large sample size, calculations were based on data for all survey years for which relevant questions were asked. The overall sample base for each condition is summarised in table 1.

To capture the relative significance of the relative strength of demographic and class gradients, we have developed a measure that we term the "relative class effect". This is defined as:

$$
\mathrm{RCE}=\left(\Sigma\left|\mathrm{P}_{\mathrm{ij}}-\mathrm{MP}_{\mathrm{j}}\right|\right) /\left(\Sigma\left|\mathrm{P}_{\mathrm{ij}}-\mathrm{MP}_{\mathrm{i}}\right|\right)
$$

where $\mathrm{P}_{\mathrm{ij}}$ is the prevalence of a particular condition in a cell defined by age category (i) and class ( $\mathrm{j}$ ) and MP is the mean prevalence for the relevant category of age or class respectively. The measure is analogous to the location quotient widely used in quantitative studies of regional economic diversity. We calculated the RCE using age and class mean prevalence rates that were both weighted and unweighted with respect to the denominator size of the cell populations.
Essentially, the RCE is the ratio of the amount that class specific prevalence rates would have to change to leave all social classes with an equal prevalence rate, compared with the extent to which age specific prevalence rates would have to change to leave all age groups with an equal prevalence rate. If the measure is greater than unity, then the social class gradient is greater in absolute terms than the demographic gradient. The lower the measure, the less significant the condition's social class gradient relative to its demographic gradient, notwithstanding the absolute magnitude of that condition's social class gradient. Conventional confirmatory significance testing is not traditionally undertaken with analogous exploratory statistical measures such as the location quotient. Instead an established "rule of thumb" takes location quotients with values between 0.75 and 1.25 to be indicative of inconclusive evidence; we take this perspective in our interpretation below.

\section{RESULTS}

We reiterate our focus on conditions that exhibit a strong statistically significant association between low social class and high prevalence. Figure 1 provides an exploratory graphical illustration of our contentions concerning the relative strength of demographic and class gradients for three marker conditions: self reported cardiac, mental, and general ill health.

The varying balance between demographic and social class factors is clear. The prevalence of grade 2 angina symptoms has a strong social gradient (particularly with respect to patients aged between 45 and 75), but also a marked demographic dimension in which prevalence generally increases with age. The known reduced importance of class based inequality in young populations is clearly evident for both sexes. The prevalence of self reported mental disorder also shows both a demographic and a social gradient. The social gradient appears less marked for women while the age gradient appears to peak in the 45-54 year group. General health seems less differentiated by class and age among women but men exhibit a ideal typical graph that peaks for older people of low social class.

Table 2 reports the values of the RCE measure for the full range of selected conditions using both the weighted and the unweighted approaches. Self assessments of mental illness and the presence of grade two angina symptoms were the only conditions where weighting with respect to the denominator size of the cell populations suggested made anything more than a negligible difference to our results.

The practical significance of class gradients varies in relation to the demographic gradient from one condition to

\begin{tabular}{|c|c|c|c|c|}
\hline & \multicolumn{2}{|l|}{ Men } & \multicolumn{2}{|l|}{ Women } \\
\hline & Total sample & Total with condition & Total sample & $\begin{array}{l}\text { Total with } \\
\text { condition }\end{array}$ \\
\hline General health "very bad" & 35541 & 439 & 41133 & 453 \\
\hline General health "bad" & 35541 & 1465 & 41133 & 1629 \\
\hline $\begin{array}{l}\text { Mental illness/anxiety/ } \\
\text { depression/nerves }\end{array}$ & 35541 & 589 & 41133 & 880 \\
\hline $\begin{array}{l}\text { Angina (grade } 2 \text { symptoms } \\
\text { reported) }\end{array}$ & 17605 & 198 & 20177 & 157 \\
\hline $\begin{array}{l}\text { Angina (grade } 1 \text { symptoms } \\
\text { reported) }\end{array}$ & 17605 & 363 & 20177 & 525 \\
\hline $\begin{array}{l}\text { Stroke/cerebral haemorrhage/ } \\
\text { cerebral thrombosis }\end{array}$ & 35541 & 298 & 41133 & 248 \\
\hline Diabetes & 35541 & 896 & 41133 & 748 \\
\hline Poor hearing/deafness & 35541 & 659 & 41133 & 479 \\
\hline Longstanding illness & 35541 & 14598 & 41133 & 17057 \\
\hline
\end{tabular}



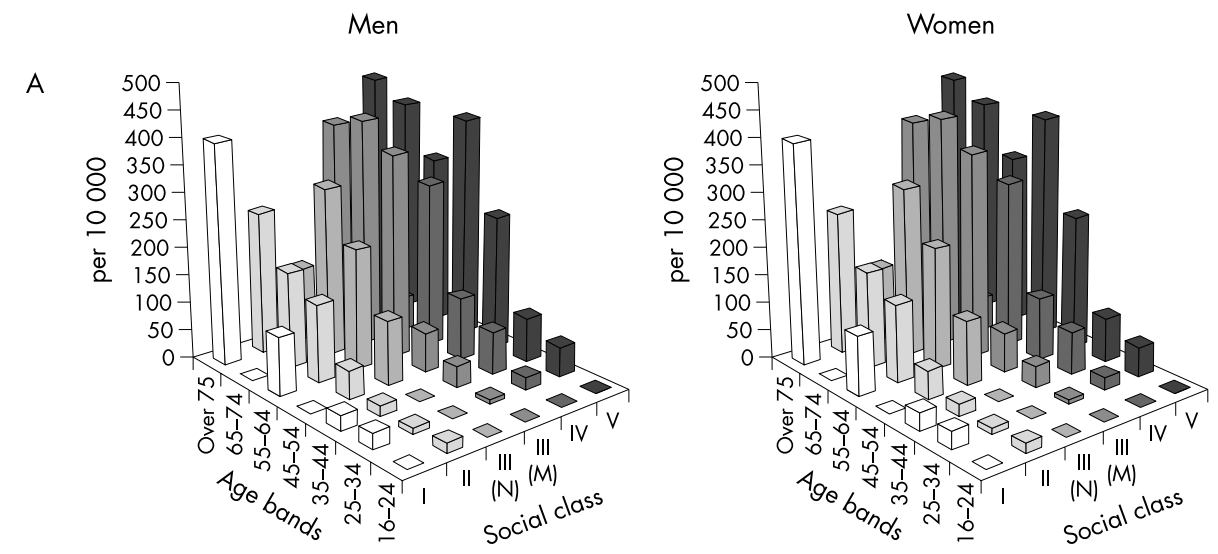

Figure 1 Age-class prevalence rates for selected conditions from the Health Survey for England, 1991-97. (A) Grade 2 angina symptoms. (B) Mental disorder (self reported). (C) Self reported "very bad health".

B

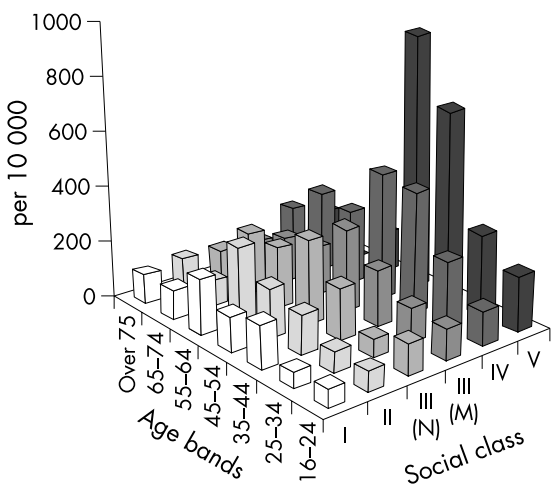

C

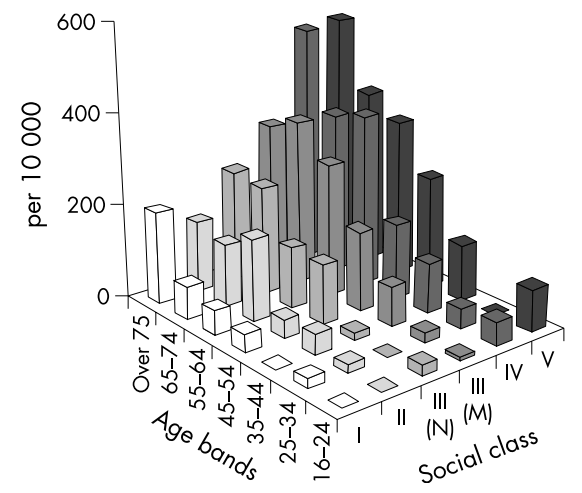

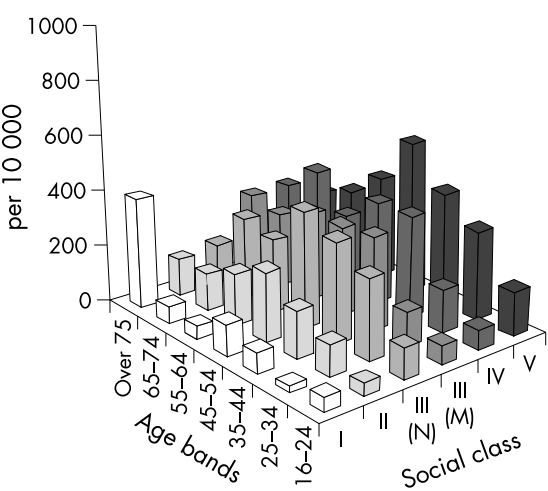

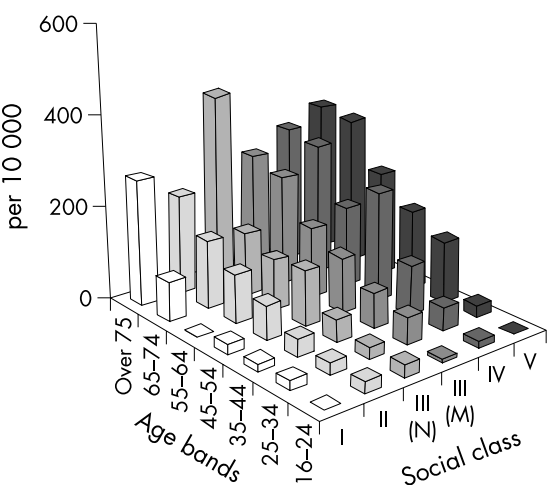

the next. Though we chose to focus on conditions that exhibited strong associations between condition prevalence and low social class, only one of condition had RCE values greater than unity. In other words, it is very unusual to find conditions where the social class gradient is greater than the demographic gradient. The sole exception to this pattern was mental illness; interestingly unweighted data suggest the dominance of class inequality among men, while weighted data suggest this happens with women. In so far as conclusions can be drawn from the application of "rules of thumb" and the observed RCE coefficients, it is unclear whether age or class dominates variations in self reported mental health. For all other conditions, age is clearly the important factor.

The RCE values display interesting variations between the sexes. Focusing on the weighted data, self reported grade 1 angina symptoms show a stronger class gradient among women but men exhibit a stronger class gradient for grade 2 symptoms. Class, though lacking the overall importance of age, is a stronger influence on male self reported health, mental health and grade 2 angina symptoms than it is on women's self assessments of the same conditions. In contrast, the self assessment of grade 2 angina symptoms,

\section{Key points}

- The age-sex component of variations in morbidity is often neglected in comparison with associated with socioeconomic status

- The magnitude of the age-sex effect on variations in self reported morbidity usually exceeds that attributable to social class in data drawn from the Health Survey for England. 


\begin{tabular}{|c|c|c|c|c|}
\hline & \multicolumn{4}{|c|}{ Relative class effect } \\
\hline & \multicolumn{2}{|c|}{ Unweighted } & \multicolumn{2}{|c|}{ Weighted } \\
\hline & Men & Women & Men & Women \\
\hline General health "very bad" & 0.677 & 0.554 & 0.672 & 0.527 \\
\hline General health "bad" & 0.736 & 0.670 & 0.749 & 0.662 \\
\hline $\begin{array}{l}\text { Mental illness/anxiety/ } \\
\text { depression/nerves }\end{array}$ & 0.892 & 1.004 & 1.008 & 0.975 \\
\hline $\begin{array}{l}\text { Angina (grade } 2 \text { symptoms } \\
\text { reported) }\end{array}$ & 0.677 & 0.477 & 0.507 & 0.447 \\
\hline $\begin{array}{l}\text { Angina (grade } 1 \text { symptoms } \\
\text { reported) }\end{array}$ & 0.500 & 0.775 & 0.493 & 0.771 \\
\hline $\begin{array}{l}\text { Stroke/cerebral haemorrhage/ } \\
\text { cerebral thrombosis }\end{array}$ & 0.290 & 0.393 & 0.275 & 0.394 \\
\hline Diabetes & 0.283 & 0.416 & 0.280 & 0.415 \\
\hline Poor hearing/deafness & 0.401 & 0.456 & 0.367 & 0.460 \\
\hline Longstanding illness & 0.177 & 0.164 & 0.165 & 0.166 \\
\hline
\end{tabular}

cerebrovascular and diabetic health and hearing problems generally has a stronger class dimension among women. These findings raise questions about variations in self reporting between sexes.

There are also similarities in our findings. Class is consistently most important for psychiatric ill health for both sexes and least important for longstanding illness. The relative ordering of conditions in terms of the (un)importance of class is similar between sexes; only in the case of angina symptoms are their clear differences. In terms of the absolute size of this "gender gap", it is most evident with respect to male self reports of very poor health and female self reports of grade one angina symptoms, diabetes, and cerebrovascular problems.

Taken as a whole, the evidence of the three dimensional graphs and the RCE statistics suggests that the case for social class inequalities for conditions in this analysis has been overstated at the expense of age. Given the underlying numerator-deonominator sizes evident in table 1 , we place least credence on our findings concerning grade 2 angina symptoms but, none the less, the interpretation that flows from our research is that the age related aspects of mortality gradients generally overshadow the social class dimensions for both sexes.

\section{DISCUSSION}

Why does this matter? Evidence from a large number of studies has consistently demonstrated that profound social gradients exist in disease prevalence. It is undeniable that many negative health conditions are more concentrated among lower social classes. We do not dispute this evidence. We do however commend a more critical analysis for two main reasons.

\section{Policy implications}

- Policies seeking to combat health inequalities need to ensure substantial sensitivity to age and gender.

- National and local initiatives targeting the health of lower socioeconomic groups, and by extension socially deprived areas, may require modification to ensure effective consideration of gender and age differentiated needs.
Firstly, there is a danger that the strong associations between class and morbidity can evolve to a simplistic suggestion that class alone is a valid indicator of poor health. Our measure of the relative class effect demonstrates however, that for both men and women many conditions, though clearly exhibiting significant social gradients, are dominated by an underlying demographic gradient. As a result, a reliance on social status alone as a surrogate for poor health is likely to over-estimate levels of poor morbidity in lower social status populations and, conversely, make underestimates for more elderly populations.

Secondly, our analysis highlights the danger of assuming that inequalities in morbidity have a consistent and universal form. Inevitably there is little scope for generalisation and substantial variation in both the age related distributions of different diseases and the degree to which different areas of morbidity are associated with socioeconomic status. Thus, while stronger age gradients may typify most conditions, some conditions are characterised by strong social gradients. From this it follows that socioeconomic disadvantage as a surrogate for health need may be appropriate for conditions that are strongly associated with deprivation, but it will be a poor proxy where that association is not strong, or where the age effect is significantly larger than the social effect.

How do these findings come about? It is clear from past work that the impact of class on morbidity is weakest in younger age groups. ${ }^{28}{ }^{29}$ Equally, in older age groups, a stronger association with age confounds the association with class. There are two immediate interpretations that can be made of the health-class-age association. Firstly, and most straightforwardly, it can be taken as an indication that older people, not unexpectedly, suffer poorer health and that differences in class background do not generate significant variations in what is overwhelmingly a process driven by aging. This process is compounded by the multifactorial and chronic nature of much ill health among all older people, age related bodily degeneration, and lower expectations of good health.

The alternative explanation is an artefactual one: while age-health associations are as undeniable as class-health ones, the confounding class-age association is problematic. Class, in the context used in this paper, is a construct derived from occupational status. Though class clearly impacts on matters such as life expectancy, its appropriateness as a measure of the socioeconomic status of post-working age people is doubtful. Our decision to use the standard occupationally based UK "class" measure of socioeconomic status in our analysis perhaps not unexpectedly highlights 
the insufficiency of that measure as a basis for the examination of health variations among older people. Without detailed longitudinal histories of changing class positions, further speculation is difficult. However, it might be hypothesised that, though occupational status is generally maximised at an age comparatively close to retirement, the positive effects of this are countered by the increasing impact of chronic disease.

In view of the condition-specific insights that are necessary to pursue key UK health policy initiatives such as the National Service Frameworks, and the additional implications of measures such as equity auditing, our analysis underlines the need to consider age as well as class when assessing inequalities in morbidity. It might be argued that this task is accomplished by comparing age standardised morbidity rates across social classes. Our results suggest that such an approach runs the risk of devaluing demography in so far as the standardisation process may remove from subsequent analysis demographic variations that appear to be often much larger than those that can be ascribed to social class. We need to take account of the relative importance of age, sex, and class. Unless we do so, a commendable concern for class based inequality may inadvertently obscure age (and gender) inequality.

\section{ACKNOWLEDGEMENTS}

We thank Theresa Lowndes for conducting a preliminary literature search in support of this study and for extracting base prevalence rates from the Health Survey for England.

\section{Authors' affiliations}

S Asthana, P Brigham, Department of Social Policy and Social Work, University of Plymouth, Plymouth, UK

A Gibson, Department of Geography, University of Exeter, Exeter, UK G Moon, School of Social and Historical Studies, University of Portsmouth, UK

J Dicker, Information Management and Technology, Swansea, UK

Funding: this work was funded by the Economic and Social Research Council's Health Variations Programme (reference L128251031).

Conflicts of interest: none declared.

\section{REFERENCES}

1 Shaw M, Dorling D, Gordon D, et al. The widening gap: health inequalities and policy in Britain. Bristol: The Policy Press, 1999:267.

2 Acheson D. Independent inquiry into inequalities in health. London: The Stationery Office, 1998:164.

3 Eachus J, Williams M, Chan P, et al. Deprivation and cause-specific morbidity: evidence from the Somerset and Avon Survey of Health. BMJ 1996:312:287-92.

4 Saul C, Payne N. How does the prevalence of specific morbidities compare with measures of socio-economic status at small area level? J Public Health Med 1999;21:340-7

5 Drever F, Whitehead M. Health inequalities: decennial supplement. London: Office for National Statistics, 1997:257.
6 Colhoun H, Prescott-Clarke P, eds. Health Survey for England, volume 1: findings. London: The Stationery Office, 1996:450.

7 Kind P, Dolan P, Gudex C, et al. Variations in population health status: results from a United Kingdom national questionnaire survey. BMJ 1998;316:736-41.

8 Payne J, Coy J, Milner P, et al. Are deprivation indicators a proxy for morbidity? A comparison of the prevalence of arthritis, depression, dyspepsia, obesity and respiritory symptoms with unemployment rates and Jarman scores. J Public Health Med 1993;16:113-14.

9 Stansfield S, Head J, Marmot M. Explaining social class differences in depression and well-being. Social Psychiatry Psychiatr Epidemiol 1998;33:1-9.

10 Marmot M, Davey Smith G, Stansfeld S, et al. Health inequalities amongst British Civil Servants: the Whitehall II study. Lancet 1991;377:1387.

11 Morrison C, Woodward M, Leslie W, et al. Effect of socioeconomic group on incidence of, management of, and survival after myocardial infarction and coronary death: analysis of community coronary event register. BMJ 1997;314:541.

12 Davey Smith G, Hart C, Watt G, et al. Individual social class, area-based deprivation, cardiovascular disease risk factors and mortality: the Renfrew and Paisley study. J Epidemiol Community Health 1998;52:399-405.

13 Woodward M, Shewry M, Smith W, et al. Social status and coronary heart disease: results from the Scottish Heart Health Study. Prev Med 1992;21:136-48.

14 Duran-Tauleria E, Rona R. Geographical and socio-economic variation in the prevalence of asthma symptoms in English and Scottish children. Thorax 1999;54:476-81.

15 Littlejohns P, Macdonald L. The relationship between severe asthma and social class. Respir Med 1993;87:139-43.

16 Strachan D, Anderson H, Limb E, et al. A national survey of asthma prevalence, severity and treatment in Great Britain. Arch Dis Child 1994;70:174-8.

17 Meadows $\mathbf{P}$. Variation of diabetes mellitus: prevalence in general practice and its relation to deprivation. Diabet Med 1995;12:696-700.

18 Ismail A, Beeching N, Gill G, et al. Capture-recapture-adjusted prevalence rates of type 2 diabetes are related to social deprivation. $Q J$ Med 1999:92:707-10.

19 Van Loon A, Brug J, Goldbohm R, ef al. Differences in cancer incidence and mortality among socio-economic groups. Scand J Social Med 1995;23:110-20.

20 Montgomery S, Morris D, Thompson P, et al. Prevalence of inflamatory bowel disease in British 26 year olds: National Longitudinal Birth Cohort. BMJ 1998;316:1058-9.

21 Butland B, Strachan D, Lewis S, et al. Investigation into the increase in hay fever and eczema at age 16 observed between the 1958 and 1970 British birth cohorts. BMJ 1997;315:717-21.

22 Cooper $\mathbf{H}$. Investigating socio-economic explanations for gender and ethnic inequalities in health. Soc Sci Med 2002;54:693-706.

23 Matthews S, Manor O, Power C. Social inequalities in health: are there gender differences? Soc Sci Med 1999:48:49-60.

24 Arber S. Comparing inequalities in women's and men's health: Britain in the 1990s. Soc Sci Med 1997;44:773-87.

25 Arber S, Ginn J. Gender and inequalities in health in later life. Soc Sci Med 1993:36:33-46.

26 West P, Macintyre S, Annandale E, et al. Social class and health in youth: findings from the West of Scotland Twenty-07 Study. Soc Sci Med 1990;30:665-73.

27 Rahkonen O, Arber S, Lahelma E. Health inequalities in early adulthood: a comparison of young men and women in Britain and Finland. Soc Sci Med 1995;41:163-71.

28 West P. Health inequalities in the early years: is there equalisation in youth? Soc Sci Med 1997;44:833-58.

29 Ford G, Ecob R, Hunt K, et al. Patterns of class inequality in health through the lifespan: class gradients at 15,35 and 55 years in the west of Scotland. Soc Sci Med 1994;39:1037-50.

30 Borooah V. Occupational class and the probability of long-term limiting illness. Soc Sci Med 1999;49:253-66. 